\title{
Phthalocyanine-sensitized hollow ZnO spheres as an efficient visiblie-light photocatalyst for water treatment
}

\author{
Cong Liu ${ }^{1}$ and Qian Duan ${ }^{1, *}$ \\ ${ }^{1}$ School of Materials Science and Engineering, Changchun University of Science and Technology, Changchun, 130022, China
}

\begin{abstract}
A novel phthalocyanine-sensitized hollow $\mathrm{ZnO}$ spheres as an efficient visiblie-light photocatalyst had been prepared successfully. Firstly, a unique hollow $\mathrm{ZnO}$ microsphere had been prepared by a facile solvothermal method followed by calcination. Secondly, zinc-tetracarboxyl-phthalocyanine (Pc) impregnated onto the surface of hollow $\mathrm{ZnO}$ microsphere. The obtained photocatalyst $\mathrm{Pc} / \mathrm{ZnO}$ was characterized by XRD, SEM and EDS. The hollow $\mathrm{Pc} / \mathrm{ZnO}$ hierarchical nanostructure improved the electron-hole separation more effectively and $\mathrm{Pc}$ loaded on the surface of $\mathrm{ZnO}$ microsphere to enhance photocatalytic activity under visible light. In our photocatalytic experiments, the hollow $\mathrm{Pc} / \mathrm{ZnO}$ microsphere showed excellent photocatalytic performance under visible light for the removal of Rhodamine $\mathrm{B}(\mathrm{RhB})$. As a result, our work provided an effective and green photocatalyst for water treatment.
\end{abstract}

\section{Introduction}

In the modern society, various pollutants (e.g., dyes, pharmaceuticals and heavy ions), which are produced by many industries, are seriously considered as major pollutants in water pollution [1]. Meanwhile, they threaten human health and living environment. Therefore, it is urgent to find effective methods for the removal of pollutants in waste water. Based on the above inspiration, photocatalysis technology has gained great potential for the elimination of hazardous waste from water environment [2].

As an important semiconductor photocatalyst, $\mathrm{ZnO}$ is intensively favored for its non-toxicity and cheapness, especially hollow structure nanoparticle. Hollow $\mathrm{ZnO}$ microsphere with adjustable morphology and size has attracted more attentions due to its more active sites and larger surface areas [3]. Nevertheless, the band gap of bare $\mathrm{ZnO}$ is $3.20 \mathrm{eV}$, which limits the application of materials to visible light region [4]. Moreover, the rapid hole-electron recombination of $\mathrm{ZnO}$ will reduce the photocatalytic performance [5]. Therefore, among various methods to overcome above shortcomings, phthalocyanine-sensitized $\mathrm{ZnO}$ seems to be a facile way to expand absorption light range and improve the photocatalytic activity [6].

In this work, we prepared a novel organic/inorganic green photocatalyst $\mathrm{Pc} / \mathrm{ZnO}$. Hollow $\mathrm{ZnO}$ microsphere was synthesized via solvothermal and pyrolysis processes. Pc impregnated on the outer surface of $\mathrm{ZnO}$. The synergistic photocatalytic performance of $\mathrm{Pc} / \mathrm{ZnO}$ was evaluated by $\mathrm{RhB}$. Benefiting from the unique design, the obtained photocatalyst exhibited better photocatalytic activity under visible light irradiation.
Above all, this study provided a new strategy for water treatment.

\section{Experiment}

\subsection{Synthesis of Hollow ZnO Spheres}

Typically, $0.35 \mathrm{~g} \mathrm{Zn}\left(\mathrm{CH}_{3} \mathrm{COO}\right)_{2}$ and $3 \mathrm{~mL}$ ethylene glycol were added in $40 \mathrm{~mL}$ ethanol. The solution was stirred for $4 \mathrm{~h}$. Then the solution was transferred into stainless steel autoclaves and maintained at $100{ }^{\circ} \mathrm{C}$ for 12 h. Products were washed for three times with ethanol by filtration processes and dried in vacuum. Finally, hollow $\mathrm{ZnO}$ spheres obtained by thermal annealing at $400^{\circ} \mathrm{C}$ for $1 \mathrm{~h}$.

\subsection{Synthesis of $\mathrm{Pc} / \mathrm{ZnO}$}

Pc was synthesized according to literature [7]. $90 \mathrm{mg}$ $\mathrm{ZnO}$ was dispersed into $15 \mathrm{~mL}$ deionized water. $10 \mathrm{mg}$ Pc dissolved in $2 \mathrm{~mL}$ dimethyl sulfoxide. Then the solution of $\mathrm{Pc}$ was added to $\mathrm{ZnO}$ with stirring for $24 \mathrm{~h}$ at $25^{\circ} \mathrm{C}$. Following that, the product $\mathrm{Pc} / \mathrm{ZnO}$ was washed with distilled water and dried in vacuum.

\subsection{Photocatalytic experiments}

A $300 \mathrm{~W}$ xenon lamp with filter acted as visible light source. $10 \mathrm{mg} \mathrm{Pc} / \mathrm{ZnO}$ was suspended in $\mathrm{RhB}$ solution $\left(1.0 \times 10^{-5} \mathrm{~mol} / \mathrm{L}, \quad 30 \mathrm{~mL}\right)$. Before photocatalytic experiments, suspension was stirred for $30 \mathrm{~min}$ without light irradiation. Then suspension was irradiated under visible light. The concentration of $\mathrm{RhB}$ was detected by UV-Vis spectrophotometer and the absorption 
wavelength was at $554 \mathrm{~nm}$. Photocatalytic degradation efficiency (D) was calculated as follows:

$$
D=\left(C_{0}-C\right) / C_{0} \times 100 \%
$$

$\mathrm{C}$ was the concentration during photocatalytic experiments and $\mathrm{C}_{0}$ was the initial concentration of $\mathrm{RhB}$ solution.

\section{Results and discussion}

The morphology of $\mathrm{ZnO}$ and $\mathrm{Pc} / \mathrm{ZnO}$ were investigated by SEM. From Fig. 1a, the prepared hollow $\mathrm{ZnO}$ were uniform spheres with diameters around $600-800 \mathrm{~nm}$. The surface of hollow $\mathrm{ZnO}$ microsphere was rough, which consisted of large numbers of nanoparticles building blocks. Compared with $\mathrm{ZnO}$, there were obvious changes in $\mathrm{Pc} / \mathrm{ZnO}$ (Fig. 1b), which might be due to the effect of Pc attachment on structure of $\mathrm{ZnO}$. The composite photocatalyst $\mathrm{Pc} / \mathrm{ZnO}$ could still maintain a complete spherical structure.

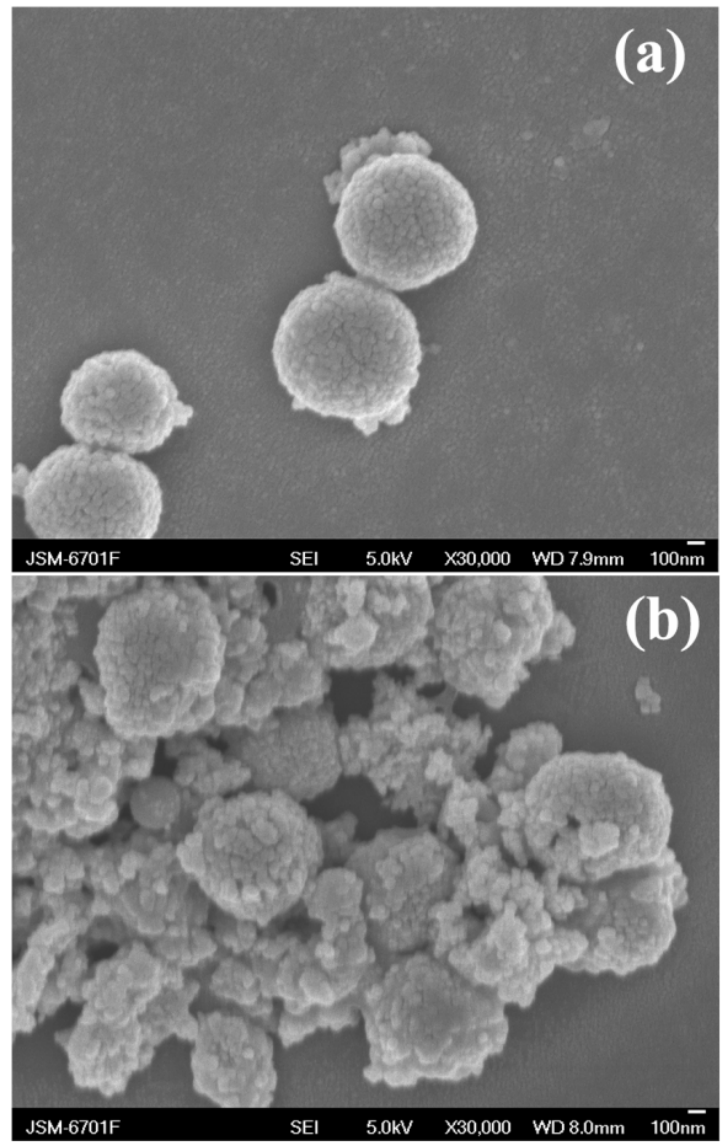

Fig. 1. SEM images of $\mathrm{ZnO}$ (a) and $\mathrm{Pc} / \mathrm{ZnO}$ (b).

The crystalline phases of $\mathrm{ZnO}$ and $\mathrm{Pc} / \mathrm{ZnO}$ were characterized by XRD in Fig. 2. The XRD pattern of $\mathrm{ZnO}$ showed diffraction peaks with high intensity and all peaks of $\mathrm{ZnO}$ matched with the JCPDS card No. 36-1451. Above results showed that the product had high crystallinity. While for $\mathrm{Pc} / \mathrm{ZnO}$, the introduction of $\mathrm{Pc}$ did not change peak positions of $\mathrm{ZnO}$, indicating synthesis process of $\mathrm{Pc} / \mathrm{ZnO}$ had little effect on the crystalline structure of $\mathrm{ZnO}$.

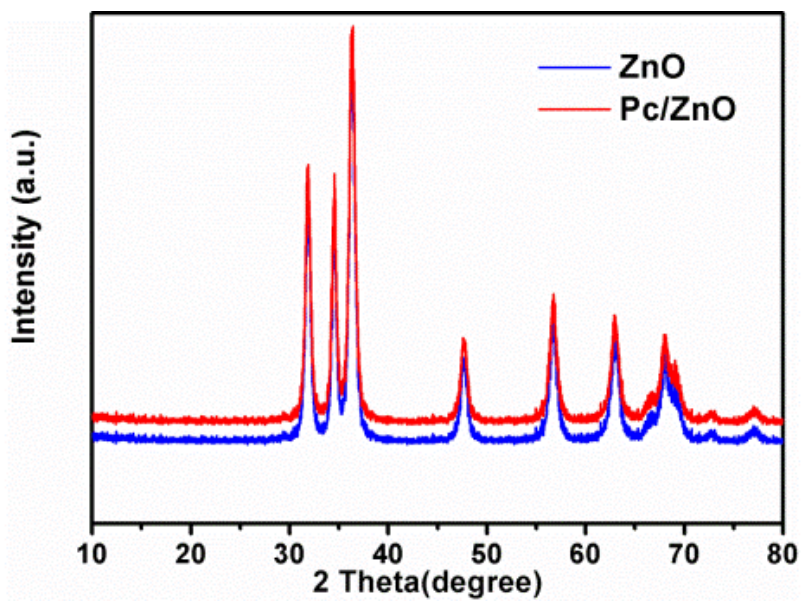

Fig. 2. $\mathrm{XRD}$ patterns of $\mathrm{ZnO}$ and $\mathrm{Pc} / \mathrm{ZnO}$.

EDS patterns of $\mathrm{ZnO}$ and $\mathrm{Pc} / \mathrm{ZnO}$ were also investigated and corresponded to above analysis. As presented in Fig. 3a, $\mathrm{Zn}$ and $\mathrm{O}$ were found from EDS spectrum and had a high content. After Pc-sensitized hollow $\mathrm{ZnO}$ spheres in Fig. 3b, the low carbon content was found, which demonstrated Pc on the surface of $\mathrm{ZnO}$. And $\mathrm{N}$ was not found which might be due to the low content.
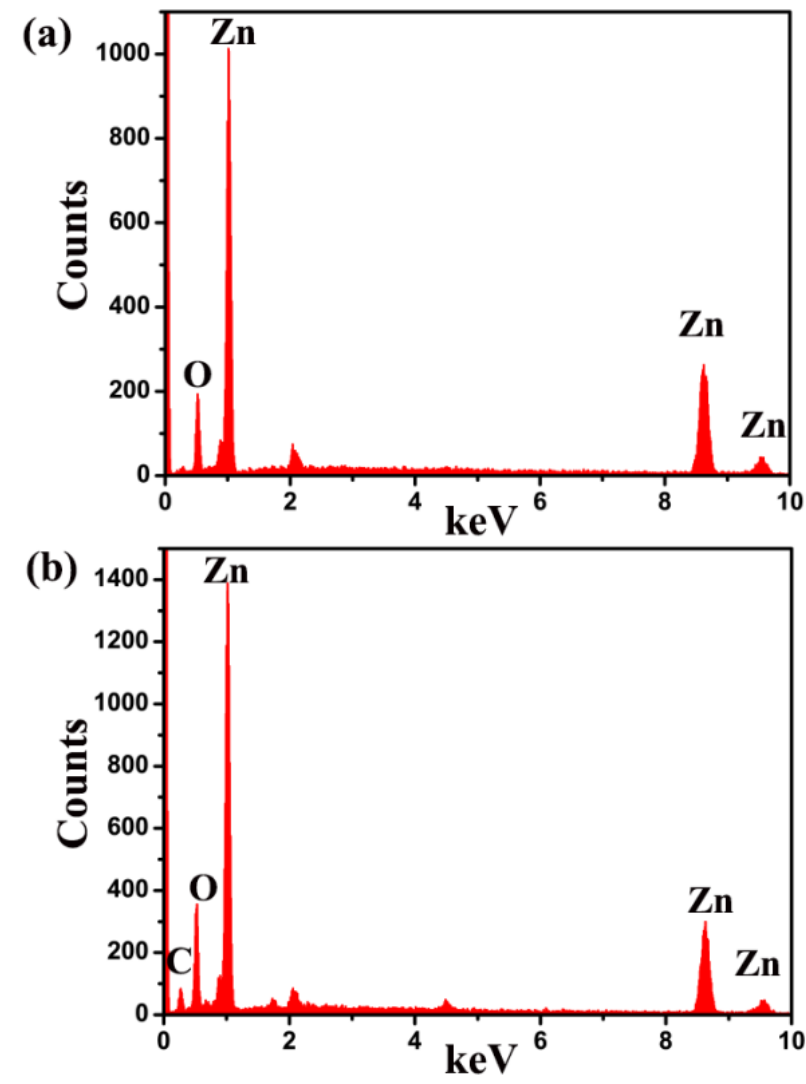

Fig. 3. EDS patterns of $\mathrm{ZnO}$ and $\mathrm{Pc} / \mathrm{ZnO}$.

To evaluate the photocatalytic activity of $\mathrm{Pc} / \mathrm{ZnO}$, $\mathrm{RhB}$ was used as model pollutants. $\mathrm{ZnO}$, due to the large band gap, was hardly to removal RhB under visible light. However, Pc sensitized $\mathrm{ZnO}$ to sufficiently make use of visible light spectrum. The photocatalytic degradation of $\mathrm{RhB}$ by $\mathrm{Pc} / \mathrm{ZnO}$ was $22 \%$ within $240 \mathrm{~min}$ under visible light irradiation showed in Fig. 4. Photocatalyst $\mathrm{Pc} / \mathrm{ZnO}$ showed excellent photocatalytic activity. 


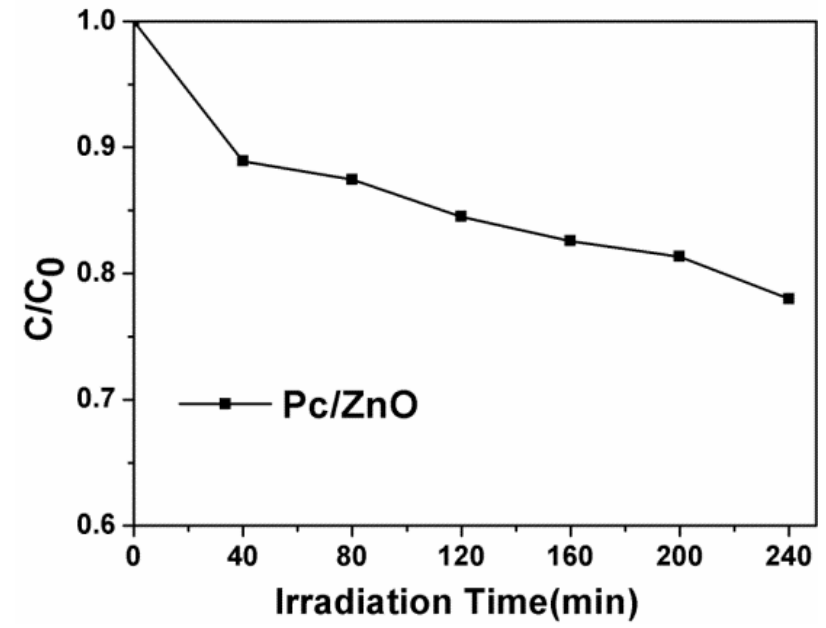

Fig. 4. The degradation curve of $\mathrm{RhB}$ by $\mathrm{Pc} / \mathrm{ZnO}$ under visible light irradiation.

\section{Conclusion}

In summary, a novel organic/inorganic green photocatalyst $\mathrm{Pc} / \mathrm{ZnO}$ was prepared. Hollow $\mathrm{ZnO}$ microsphere was synthesized via solvothermal and pyrolysis processes. Then $\mathrm{Pc}$ impregnated on the surface of $\mathrm{ZnO}$. Pc sensitized hollow $\mathrm{ZnO}$ to improve its light response to visible region, which improved photocatalytic activity. Thanks for the reasonable structural design, the efficiency of photocatalyst was improved under visible light. Therefore, this study provided a deeper investigation on hollow $\mathrm{Pc} / \mathrm{ZnO}$ microsphere, which would be an ideal photocatalyst in water treatment.

\section{Acknowledgments}

We would like to thank the National Natural Science Foundation of China (21153003), Jilin Science \& Technology Department (20190303069SF, $20150520025 \mathrm{JH}$ ), Changchun University of Science and Technology Innovation Foundation (XJJLG-2016-06, XJJLG-2016-05).

\section{References}

1. B. Wang, Z. Xie, Y. Li, Z. Yang, L. Chen, Macromolecules. 51, 3443-3449 (2018).

2. C. Li, Z. Sun, W. Zhang, C. Yu, S. Zheng, Appl. Catal. B: Environ. 220, 272-282 (2018).

3. Y. Li, S. Wang, P. Hao, J. Tian, H. Cui, X. Wang, Sens. Actuators B. 273, 751-759 (2018).

4. D. Ma, J.-W. Shi, D. Sun, Y. Zou, L. Cheng, C. He, H. Wang, C. Niu, L. Wang, Appl. Catal. B: Environ. 244, 748-757 (2019).

5. L. Cao, C. Yang, B. Zhang, K. Lv, M. Li, K. Deng, J. Hazard. Mater. 359, 388-395 (2018).

6. M.L. Maya-Treviño, J.L. Guzmán-Mar, L. Hinojosa-Reyes, Mater. Sci. Semicond. Process. 77, 74-82 (2018).
7. C. Chen, Z. Ma, S. Zhou, T. Li, X. Sun, Catal. Lett. 147, 2399-2409(2017). 